\title{
Performance Analysis of Color based Image Retrieval
}

\author{
Metty Mustikasari \\ Information System Department - Gunadarma University \\ metty@staff.gunadarma.ac.id \\ Sarifuddin Madenda
}

High School of Computer Science and Management Jakarta (STMIK JAKARTA)

sarif@jak-stik.ac.id

\begin{abstract}
Recently Content based image retrieval (CBIR) is an active research. This paper proposes a technique to retrieve images based on color feature and evaluate the retrieval system performance. In this retrieval system Euclidean distance and City block distance are used to measure similarity of images. This algorithm is tested by using Corel image database which is provided by James Wang. The performance of retrieval system is measured in terms of its recall and precision. The effectiveness of retrieval system is also measured based on Average Rank (AVRR) of all relevant retrieves images and Ideal Average Rank of relevant images (IAVRR). The experimental results show that city block has achieved higher retrieval performance than Euclidean distance.
\end{abstract}

Keywords: Content-based image retrieval (CBIR), color histogram, color spaces, Euclidean distance, City block distance, precision, recall.

\section{Council for Innovative Research}

Peer Review Research Publishing System

Journal: INTERNATIONAL JOURNAL OF COMPUTERS \& TECHNOLOGY

Vol. 12, No.4

editor@cirworld.com

www.ijctonline.com, www.cirworld.com 


\section{INTRODUCTION}

Content-based image retrieval (CBIR) has become an important research topic because of propagation of digital images. Image retrieval systems attempt to search through the database to find images that similar to the query image. CBIR is an alternative to traditional text-based image search, and can improve the accuracy of the information that is returned. It is an efficient technique for visual content-based search and a process of retrieving images from a library of digital images according to the visual content of images.

CBIR process consists of computing a feature vector that characterizes the nature of the images. The images are stored in a feature database. In CBIR system the user provides a query image and the system calculates the feature vector, and then compares it with a certain image features of the image database. Comparisons were made by using a distance measurement technique, and the minimum distance is a metric for the matched or similar images. Feature vectors should be capable enough to fully characterize the structural and spatial image properties, which take the same image from the image database.

Color is one of the most powerful visual features and widely used in image retrieval because is easier to implement in image retrieval system. Color is independent of image size and orientation, because it is robust to background complication. Color histogram is the most common technique for extracting color image features because of the calculation of the low cost and is not sensitive to small variations in the structure of the image. However, color histogram holds two major drawbacks. They can not fully accommodate the spatial information, and they are not unique. Two different images with the same distribution of color histogram is very similar result. In addition, the same images from the same viewpoint carrying different lighting conditions make different histogram.

Currently a lot of research Gathering Recognize the content based image retrieval using color features. Jeong proposed color based image retrieval using histogram approach [3]. They investigated RGB color space and HSV color space using Euclidean, histogram intersection and quadratic distance. In general histogram based retrievals in HSV color space showed better performance than in RGB color space since it showed higher precision values for the same recall values. In addition using histogram intersection method is faster than using Euclidean or quadratic method. Singha and Hemachandran analyzed the performance of color based image retrieval to identify the various color space (Lab, RGB, HSV, LUV) [10]. The color spaces were investigated using Histogram Intersection. The evaluation of retrieval systems performance is measured using precision and recall. In their experiment HSV color space gives the best performance than other.

This paper describes a color based image retrieval system and comparison of the retrieval performance. The rest of the paper consists of: Section II presents the proposed method. Section III describes the experimental results. Finally, Section IV presents the conclusions.

\section{PROPOSED METHOD}

\subsection{Method}

The outline of the method in this experiment is described as follows:

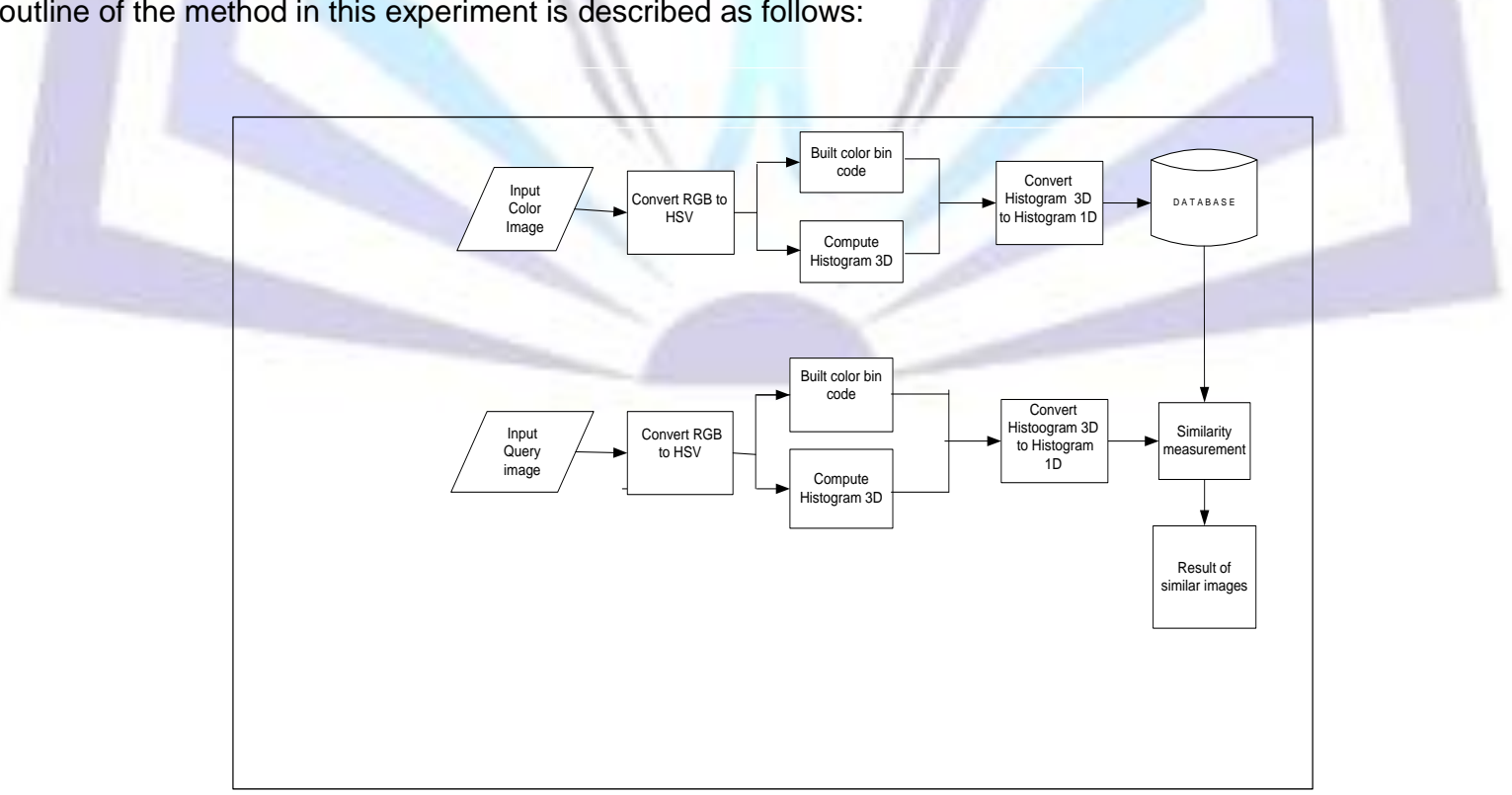

Figure 1. Proposed Method scheme 
In the Figure 1, a method scheme of color based image retrieval is proposed. Once a query image is chosen by users, the process is done automatically. There are two stages of image retrieval. The first one is image storing and the second is image retrieval. In the first stage, while image query is chosen, the RGB color space is converted to HSV color space. After that process is conducted by the system, color bin code is built. While the process of color bin code is done, $3 \mathrm{D}$ histogram is computed. After the histogram is computed then the $3 \mathrm{D}$ histogram is converted to $1 \mathrm{D}$ histogram. All the images are saved to the database. The second stage has the same process with the first stage. After converting the Histogram $3 D$ to histogram $1 D$, similarity measurement between query image and database image is computed using Euclidean distance or city Block distance then the result is ordered.

\subsection{Color Space}

Compared with texture and shape features, color feature is one of the most sifnificant features of image retrieval. A color space is a model for representing color in terms of intensity values. It specifies how color information is represented. Typically, a color space defines a one-to four dimensional space [3]. A color component, or a color channel is one of the dimensions.

There are many color models to express the color in an image such as RGB (Red, Green, Blue), HSL (Hue, Saturation, Luminance), HSV/HSB (Hue, Saturation, Value or Brightness), HSI (Hue, Saturation, Intensity), L* $u^{*} v^{*}$, dan $^{L^{*} a^{*} b^{\star}}$ (luminance $\mathrm{L}^{*}$, chrominance $\mathrm{u}^{*}, \mathrm{v}^{*}, \mathrm{a}^{*}$, dan $\mathrm{b}^{*}$ ), CIECAM02 dan HCL [9].

\subsection{HSV Color Spaces}

The HSV stands for the Hue, Saturation, and Value. HSV is one of several color systems which is considerably closer than RGB color space to the way of humans experience. The HSV color space is formulated by looking at the RGB color cube along its gray axis (the axis joining the black and white vertices), which result in the hexagonally shaped color palette [2].

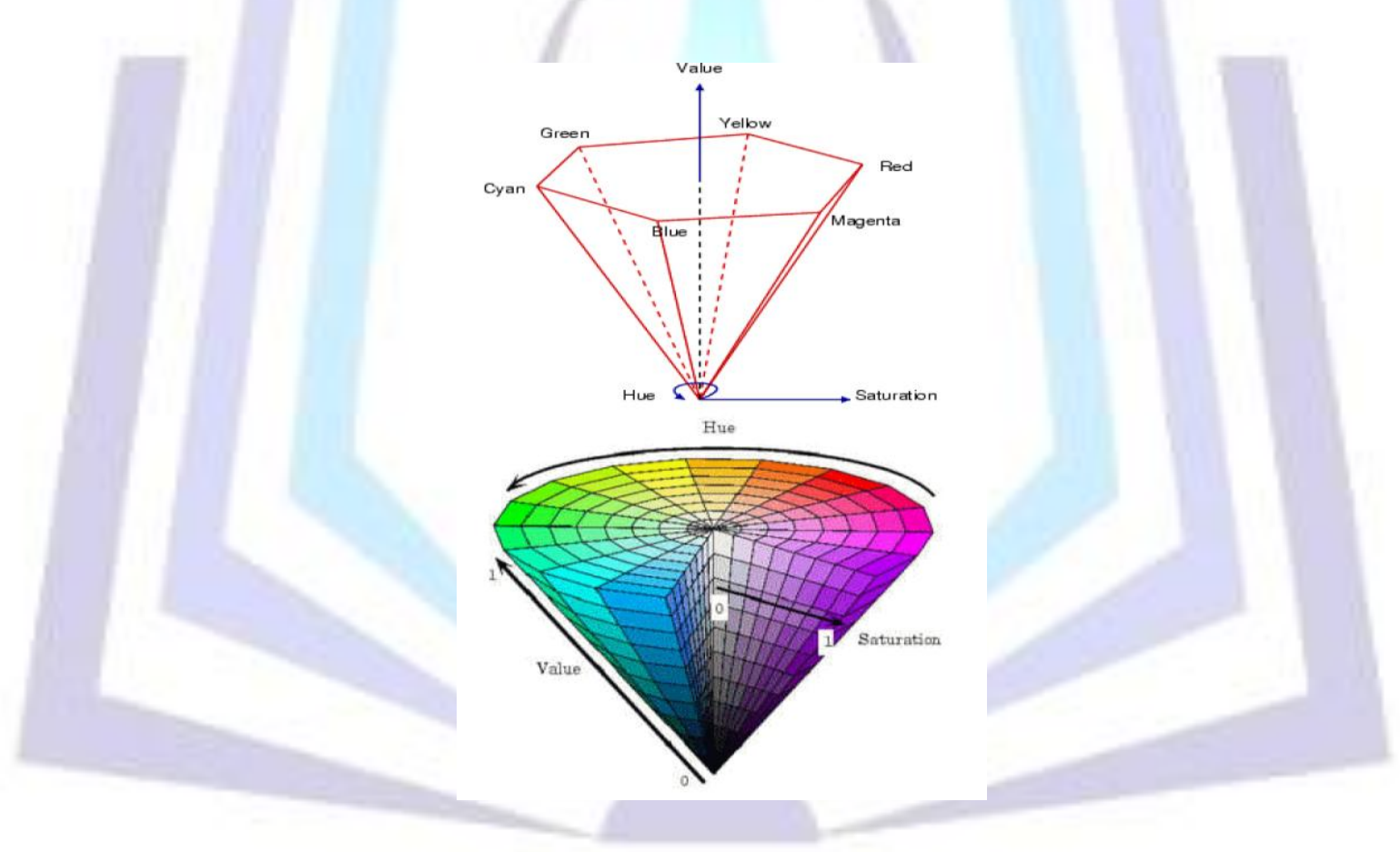

Figure 2. HSV Color space

The Value represents intensity of a color, which is decoupled from the color information in there presented image. The hue and saturation components are related to the way human eye perceives color resulting in image processing algorithms with physiological basis. As hue varies from 0 to 1.0, the corresponding colors vary from red, through yellow, green, cyan, blue, and magenta, back to red, so that there are actually red values both at 0 and 1.0 [3]. As saturation varies from 0 to 1.0 , the corresponding colors (hues) vary from unsaturated (shades of gray) to fully saturated (no white component). As value, or brightness, varies from 0 to 1.0 , the corresponding colors become increasingly brighter.

Gonzales and woods [1] use the following equations for RGB to HSV conversion:

$$
\begin{gathered}
H=\operatorname{COS}^{-1}\left\{\frac{\frac{1}{2}[(R-G)+(R-B)]}{\sqrt{\left(R-G^{2}+(R-B)(G-B)\right)}}\right\} \\
s=1-\frac{3}{R+G+B}[\min (R, G, B)], v=\frac{1}{3}(R+G+B)
\end{gathered}
$$




\subsection{Color Space Quantization}

Color space quantization is a process that reduces the number of distinct colors employed to represent an image, usually with the intention that the new image should be as visually similar as possible to the original image. The number of the kind of colors for a true color image are up to $2^{24}=16777216$, therefore the direct extraction of color feature from true color will lead to a large computation. In order to reduce the computation, without a significant reduction in image quality, some representative color is extracted to represent the image, thereby reducing the storage space and enhancing the process speed [10].

A quantization scheme is determined by the color model and the segmentation of the color model used. Usually color models represent a color in the form of tuples (generally of three). By applying a standard quantization scheme to a color model, each axis is divided into a certain number of fractions. When the axes divided into three parts, number ( $n$ ) of the colors used represent an image will be multiplication of the parts. A quantization of color model in $n$ colors is often referred to as a n-bins quantization scheme.

In HSV color space, quantization of hue requires the most attention. In this research Hue is quantized to 11 level, saturations and value are each quantized to six levels yielding greater perceptual tolerance along these dimensions. Thus the quantized HSV space has $11 \times 6 \times 6=376$ histogram bins.

\subsection{Histogram Computation}

A color histogram represents the distribution of colors in an image, where each histogram bin corresponds to a color in the quantized color space [13]. It represents the number of pixels that have colors in each of a fixed list of color ranges that span the images's color spaces, the set of all possible colors. Color histograms are a set of bins, where each bin denotes the probability of pixels in the image being of a particular color. A color histogram for a given image is defined as a vector:

$$
H=\{H[0], H[1], H[2], \ldots, \ldots, H[i], \ldots, H[n]\}
$$

Where i represents a color in the color histogram and $\mathrm{H}[\mathrm{i}]$ is the number of pixels in color $\mathrm{i}$ in the Image, and $\mathrm{n}$ is the total number of bins used in color histogram. Typically, each Implementation pixel in an image will be assigned to a bin of a color histogram of that image, therefore for the color histogram of an image, the value of each bin is the number of pixels that has the same corresponding color. In order to compare images of different sizes, color histograms should be normalized. The normalized color histogram $\mathrm{H}^{\prime}$ is defined as:

$$
H^{\prime}=\left\{H^{\prime}[0], H^{\prime}[1], H^{\prime}[2], \ldots \ldots, H^{\prime}[i], \ldots ., H^{\prime}[n]\right\}
$$

Where $\mathrm{H}^{\prime}[\mathrm{i}]=\frac{H[i]}{P}, \mathrm{p}$ is the total number of pixels of an image.

\subsection{Distance Metrics}

\subsubsection{City block Distance}

City block distance is called as Manhattan distance. City block distance method calculates the distance value of 2 vectors. The Formula of the method is as follow:

$$
\mathrm{d}(q, b)=\sum_{\mathrm{h}=1}^{\mathrm{H}} \quad \sum_{\mathrm{s}=1}^{\mathrm{S}} \quad \sum_{\mathrm{v}=1}^{\mathrm{V}} \quad(|\mathrm{q}(\mathrm{h}, \mathrm{s}, \mathrm{v})-\mathrm{b}(\mathrm{h}, \mathrm{s}, \mathrm{v})|)
$$

This distance formula represents computation of the distance between query image and database image. Where $q(h, s, v)$ represents coordinate color bin of query image and $b(h, s, v)$ represents coordinate color bin of database image.

The City block distance is always greater than or equal to zero. The measurement would be zero for identical points and high for points that show little similarity.

\subsubsection{Euclidean distance metrics}

The Euclidean Distances is one of method is used for measure similarity of image retrieval [3]. Euclidean distance can be treated as the spatial distance in a multi-dimensional space. In this research, we used the square root of Euclidean distance, which is defined as:

$$
\mathrm{d}^{2}(q, b)=\sum_{\mathrm{h}=1}^{\mathrm{H}} \quad \sum_{\mathrm{s}=1}^{\mathrm{S}} \quad \sum_{\mathrm{v}=1}^{\mathrm{V}} \quad(\mathrm{q}(\mathrm{h}, \mathrm{s}, \mathrm{v})-\mathrm{b}(\mathrm{h}, \mathrm{s}, \mathrm{v}))^{2}
$$

This distance formula represents computation of the distance between query image and database image. Where $q(h, s, v)$ represents coordinate color bin of query image and $b(h, s, v)$ represents coordinate color bin of database image.

\subsection{Performance Evaluation}

\subsubsection{Recall and Precision}

The performance of retrieval system can be measured by metrics such as recall and precision [1]. Those metrics can be measured the effectiveness of the image retrieval. As Precision and Recall are a standard way to evaluate retrieval results for image retrieval systems, hence we used them in our research. 
Recall signifies the relevant images in the database that retrieves in response to a query. Precision is the proportion of the retrieved images that are relevant to the query. More precisely, let $A$ be the set of relevant items, let $B$ the set of the retrieved items and a, b, c are given in Figure 3.

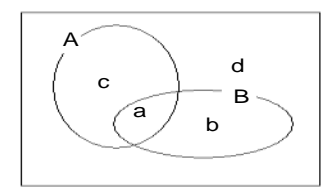

Figure 3. Sets for explaining retrieval effectiveness

In figure 3, a stands for 'retrieved relevant' images, $b$ for 'retrieved irrelevant' images, $c$ for 'unretrieved relevant' images and $d$ for 'unretrieved irrelevant' images. Then recall and precision are defined as the following conditional probabilities [3].

$$
\begin{aligned}
& \text { Recall }=P(B \backslash A)=\frac{\mathrm{P}(\mathrm{A} \cap \mathrm{B})}{\mathrm{P}(\mathrm{A})}=\frac{\mathrm{a}}{\mathrm{a}+\mathrm{c}} \\
& \text { Precision }=P(A \backslash B)=\frac{\mathrm{P}(\mathrm{A} \cap \mathrm{B})}{\mathrm{P}(\mathrm{B})}=\frac{\mathrm{a}}{\mathrm{a}+\mathrm{b}}
\end{aligned}
$$

With these conditions, images retrieval is said to be more effective if precision values are higher at the same recall values.

\subsubsection{AVRR and IAVRR}

Image Retrieval performance in this paper is analyzed according to its accuracy. Indicators such as precision and recall are commonly used for retrieval effectiveness computation. However, they do not really reflect the accuracy of the image retrieval system because the ranking of each displayed image is generally not taken into account. The normalized recall measure partially overcomes this limitation [7].

Faloutsos et al. have defined a measure for evaluating the effectiveness of QBIC system [7]. For each image query, the average rank (AVRR) of all relevant retrieved images is computed as well as the ideal average rank of relevant images (IAVRR). The formula assumes that the system returns all the $P$ relevant images which, in the ideal case (IAVRR), occupy the first $P$ positions. This effectiveness measure obviously takes into account the ranking of relevant images. However, it ignores the deviation between the ideal ranking and the actual ranking of a relevant image. For example, if the system returns images in a completely inverse order of the ideal ranking, the following formula returns a perfect effectiveness value $(=1)$.

$$
\mathrm{Eff}=\frac{\text { AVRR }}{I A V R R} \text {, where IAVRR }=\sum_{\mathrm{i}=1}^{\mathrm{P}} \frac{\mathrm{i}}{\mathrm{P}} \text { and AVRR }=\sum_{\mathrm{i}=1}^{\mathrm{P}} \frac{\mathrm{ri}}{\mathrm{P}}
$$

where $P$ is the total number of relevant images, $i=(1,2, \cdots, P)$ is similarity image ranking by human expert judgement and $r_{i}$ corresponds to system image ranking (in a decreasing relevance order) [7].

\section{EXPERIMENTAL RESULT}

The proposed algorithm is tested by using Corel image database which is provided by James Wang. This original database consists of 1000 images having 10 categories and each of which has 100 images. This experiment uses only 500 color images and 5 categories of Images which are beach, dinosaurs, bus, flower, and mountain. Color space is used in this experiment is HSV Color space in JPEG format. The experiment has been carried out in the Matlab environment.

The retrieval method is shown in the block diagram Figure 1. As stated before the color spaces used in this experiment is HSV. Normally the query and database images are represent in RGB color space and is converted into respective quantization as HSV (11X6X6) color pixels. For the first stage all 500 color images are saved to the database which is divided into five categories. The second stage is retrieval system that is address five queries from five categories to be retrieved.

The empirical analysis of retrieval system is conducted into two steps: (i) Image retrieval using city block and Euclidean distances, and (ii) Performance evaluation using Precision, Recall and Effectiveness computation based on participant's ranking of similar images. For the first step, five image queries from five categories were addressed to the system. City block and Euclidean distance are used to measure similarity between queries and the images present in the database. If the distance between feature vectors of the query image and feature vectors image in the databases is equal to zero the corresponding image in the database is to be considered as a match to the query. The search is based on similarity rather than on exact match and the retrieval results are then ranked accordingly to a similarity index. The second step is the retrieval performance results that are evaluated using recall, precision and effectiveness using AVRR and IAVRR. The Effectiveness is counted based on the averaged ranked of retrieval (AVRR) results divide by the Ideal Averaged ranked (IAVRR) which are ranked by participants. 
We have tested our retrieval algorithm on image database with 500 images which contain several class images such as beach, building, bus, flower, and mountain. Figure 4 represents retrieval results for category beach using City block distance whereas figure 5 represents retrieval results for category beach using Euclidean distance. When a search for the images is requested, a selection process will recover the closest images in the database. In each set, on top left corner is the query image and the retrieved image are listed according to their distance with the query image. On top of every retrieved image its distance from the query image is shown. It should be noted that in each set of retrieval, the first image retrieved is the query image itself is present in the database, it will be retrieved first indicating perfect match.

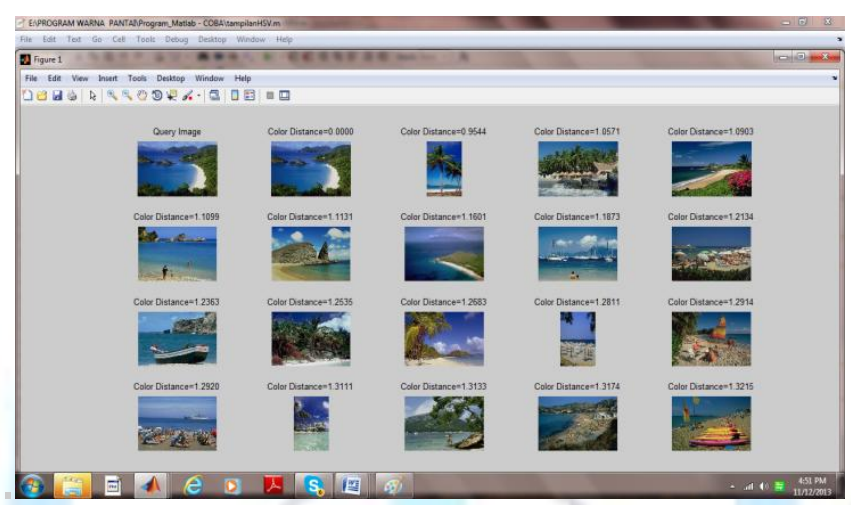

Figure 4. Image retrieval results using City block Distance

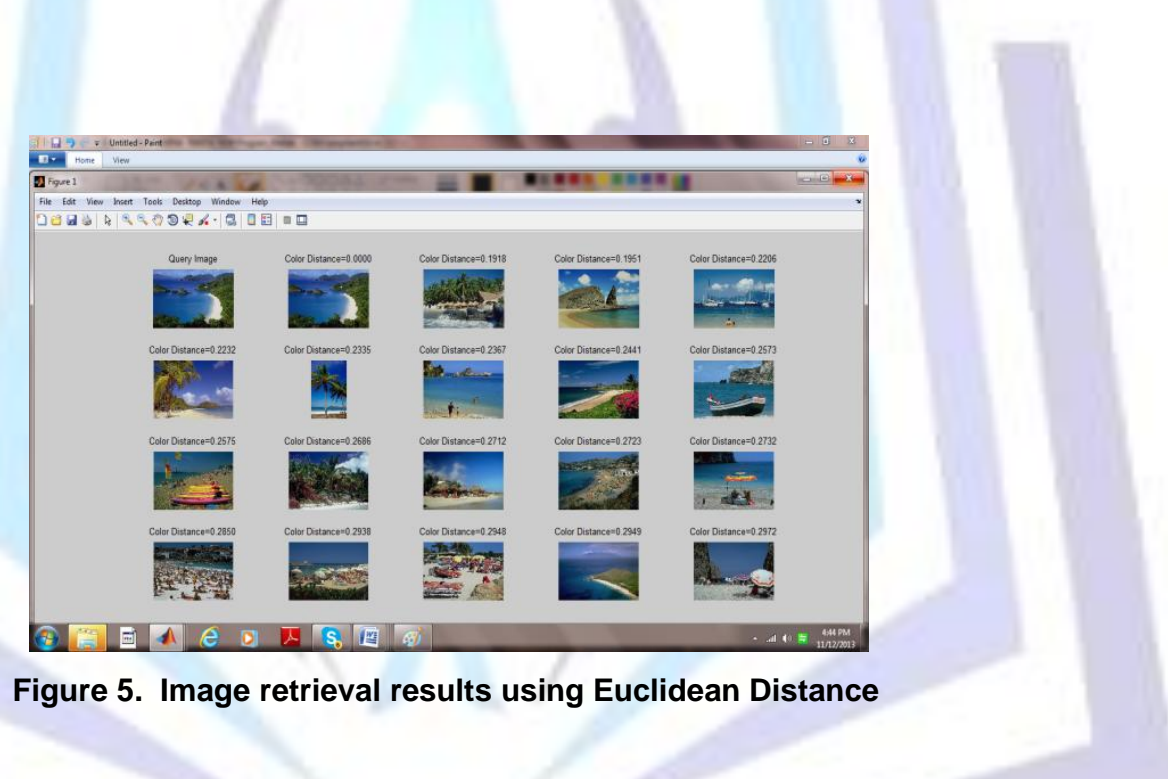

As stated before for evaluating the quality of image retrieval, we used Average Rank (AVRR), Ideal Average Rank (IAVRR) to obtain retrieval effectiveness, precision and recall parameters. The retrieval evaluation results can be shown in table 1 and figure 6. Algorithm is stated as effective if the value of effectiveness using AVRR and IAVRR is 1 . Images retrieval is also said to be more effective if precision values are higher at the same recall values. The higher the retrieval precision, the better the distance measure conforms to human perception. 
Table 1. Average Precision

\begin{tabular}{|c|c|c|}
\hline Query Image & $\begin{array}{c}\text { Precision } \\
\text { City Block } \\
\text { Distance }\end{array}$ & $\begin{array}{c}\text { Precision } \\
\text { Euclidean } \\
\text { Distance }\end{array}$ \\
\hline Beach & 0.588 & 0.555 \\
\hline Dinosaurs & 0.769 & 0.434 \\
\hline Bus & 0.588 & 0.384 \\
\hline $\begin{array}{c}\text { Flower } \\
\text { s. }\end{array}$ & 0.769 & 0.833 \\
\hline Mountain & & \\
\hline Average & $\mathbf{0 . 6 7 6}$ & $\mathbf{0 . 5 3 6}$ \\
\hline
\end{tabular}

As we can see from table 1 and figure 6 the retrieval results of 5 types of images, the range of precision using city block distance is 0.588 to 0.769 and the precision average is 0.676 . Whereas the range of precision using Euclidean distance is 0.384 to 0.833 and the precision average is 0.536 . The average precision using City block Distance is 0.676 and the average precision using Euclidean Distance is 0.536 .

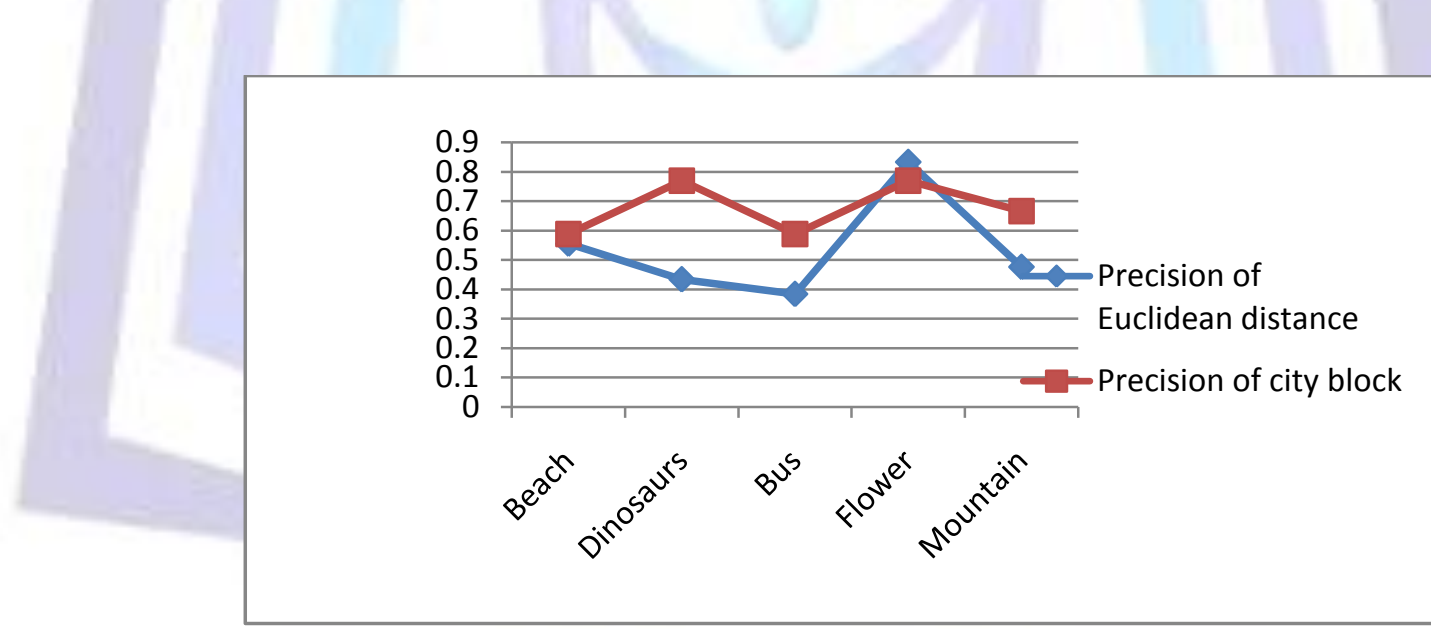

Figure 6. Precision of Euclidean and City Block distances

The retrieval results using Euclidean distance can been seen from table 2 and figure 7. The range of the value retrieval effectiveness of City block Distance is 1.072 to 1.418 and the range of the value retrieval effectiveness of Euclidean Distance is 1.107 to 1.927 . The average effectiveness using City block Distance is 1.217 and the average effectiveness using Euclidean Distance is 1.431. 
Table 2. Average Effectiveness using AVRR and IAVRR

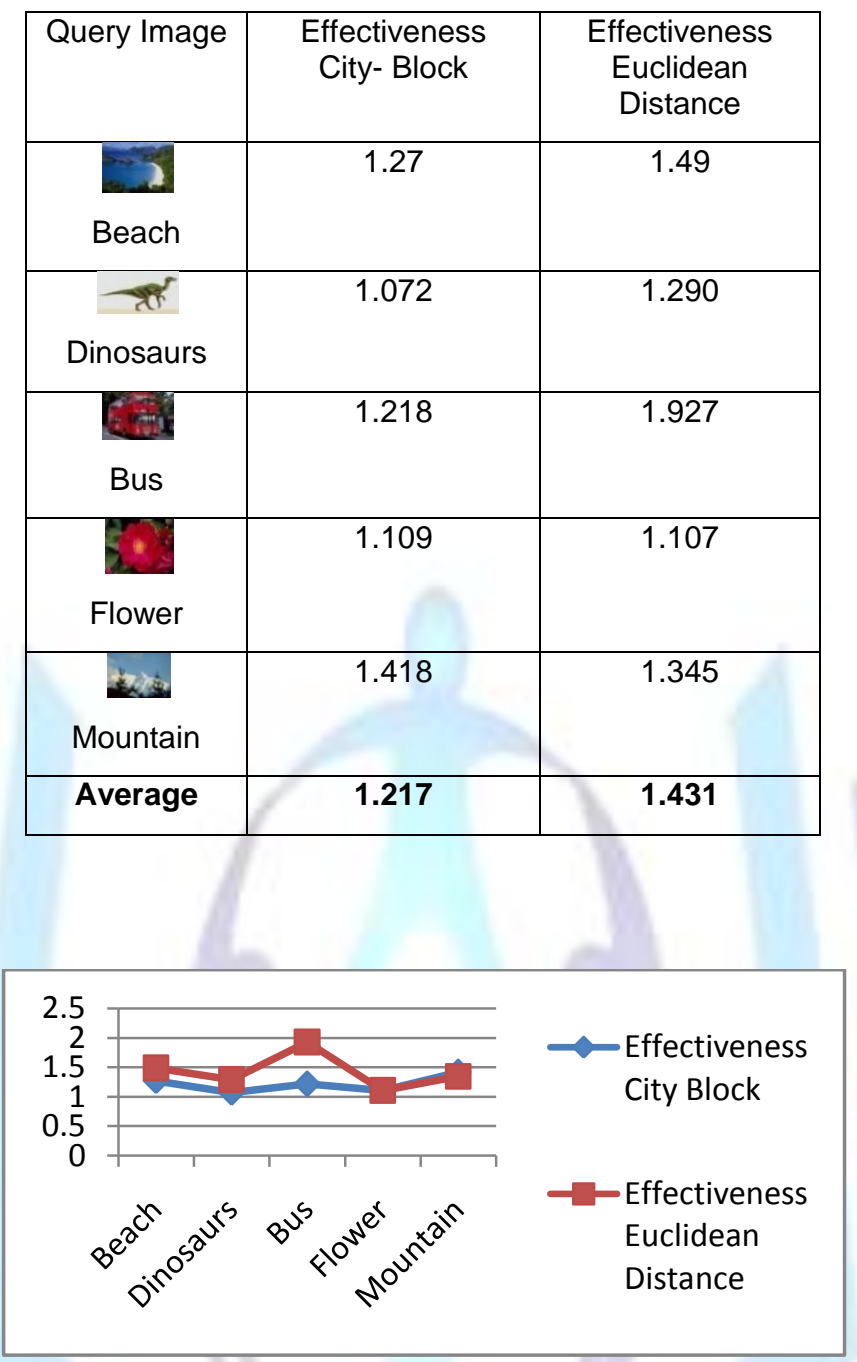

Figure 7. Effectiveness of Euclidean and City Block distances

As can be seen in figure 6 and table 1, the retrieval results for flower images using city block has the highest precision. The retrieval results for flower images using Euclidean distance also has the highest precision. The retrieval result of city block distance using AVRR and IAVRR for dinosaur images as can be seen from figure 7 and table 2 has the most effective as the value retrieval effectiveness is nearest to 1 . Whereas the retrieval results for flower images of Euclidean distance using AVRR and IAVRR has the most effective.

From the retrieval effectiveness it is found that Euclidean distance performs significantly lower than city block distance. City block distance filters out the irrelevant elements in the matching of two feature vectors. However the obtained result is depending on a combination among the number of the images to be recovered, quantization used, color space, metrics, and the threshold considered.

\section{CONCLUSION}

In this paper an image retrieval using color histogram technique is proposed. In order to compare the performance of the algorithm, two similarity measurements in image retrieval have been described and evaluated. The First similarity measurement is city block distance and the other is Euclidean distance. The Retrieval effectiveness are evaluated using recall, precision, AVRR and IAVRR. Experiment results show that in terms of retrieval effectiveness, city block distance are more desirable than Euclidean distance measurements for determining image similarity.

From the results we conclude that City block gives better performance than Euclidean distance in terms of accuracy. City block distance is simpler than Euclidean distance and it is more desirable for online retrieval. As further studies the proposed retrieval method should be evaluated for more various data and similarity measurements. 


\section{REFERENCES}

[1] Gonzalez R.C. and Woods R.E. 2009. "Digital Image Processing”. Prentice Hall Inc., New Jersey, $2^{\text {nd. }}$

[2] Gonzalez R.C., Woods R.E., Eddins S.L. 2009. "Digital Image Processing using MATLAB ". Gatesmark publishing Division of Gatemarks, LLC.

[3] Jeong S. 2001. "Histogram-Based Color Image Retrieval”. Psych221/EE362 Project.

[4] Kodikuwakku S.R., Selvarajah S. 2012. "Comparison of Color Features for Image Retrieval". Indian Journal of Computer Science and Engineering. Vol.1. No.3.PP.207-211.

[5] Komali A., et.al. 2012. "3D Color Feature Extraction in Content-Based Image Retrieval". International Journal of soft Computing and Engineering. Vol.2. Issue.3.PP.560-563.

[6] Kurhe A.B., Satonka S.S., and Khanale P.B. 2011. "Color Matching of Images by using Minkowski-Form Distance". Global Journal of Computer Science \& technology.vol.11.No.5.

[7] Missaoui R., Sarifuddin M. and Vaillancourt J. 2004. "An Effective Approach Towards Content-Based Image Retrieval". CIVR. Pp.335-343.

[8] Niranjanan S., Gopalan S.P.R. 2012. "Performance Efficiency of Quantization using HSV Colour space and Intersection Distance in CBIR". International Journal of Computer Application. Vol.42. No.21.pp.48-55.

[9] Sarifuddin M., Missaoui R. 2005. "A new Perceptually Uniform Color space with associated color similarity measure for content-based image and video retrieval". Proceedings of Multimedia Information Retrieval Workshop, $28^{\text {th }}$ annual ACM Sigir Conference. pp. 1-8.

[10] Singha M., Hemachandran K., 2011. "Performance analysis of color spaces in image retrieval". Assam University journal of science. Vol.7. No.11.

[11] Singha N., Shandila S.K. 2010. "A Survey on: Content Based Image Retrieval System". International Journal of Computer Applications. Vol.4. No.2.PP.22-24.

[12] Suhasini P.S., Krishna K.S.R., Krishna I.V.M. 2009. "CBIR Using Color Histogram Processing". Journal of Theoretical and Applied Information Technology.

[13] Wang S. 2001. "A Robust CBIR Approach using Local Color Histograms". Department of Computing Science.

[14] Xiaoling W., and Hongyan M. 2009. "Enhancing Color histogram for Image Retrieval". International Journal Workshop on Information security and Aplication.

[15] Zuva K., Zuva T. 2012. "Evaluation of Information Retrieval System". International Journal of Computer science and Information Technology.Vol.4. No.3.pp.35-43.

\section{Author's Biography}

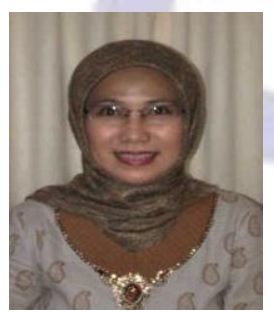

Metty Mustikasari is currently a lecturer at Information System Department, Gunadarma University. She receives her Bachelor degree from Information System Department, Gunadarma University, Indonesia in 1987 and received Master degree from Computer Science Department, Curtin University of Technology, Western Australia in 1991. She is currently a Ph.D student at the Department of Information Technology, Gunadarma University. Her research interests are image retrieval, information retrieval, database design and data mining.

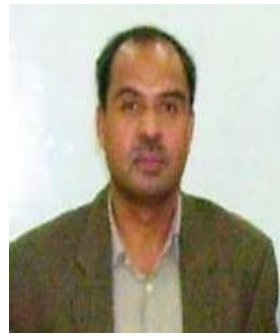

Sarifuddin Madenda is currently Director of High School of Computer Science and Management Jakarta (STMIK Jakarta STI\&K). He received the B.S. degree from University of Indonesia, in 1989, the M.S. degree from Institute National des Sciences Appliquées de Lyon (INSA de Lyon) - French, in 1992, and the Ph.D. degree from University of Burgundy - French, in 1995. From 1995 to 1996, he was a Research Associate at LIESIB Laboratory - University of Burgundy. In 1997, he became a Lecturer in Computer Science Department, Gunadarma University - Indonesia. From 2002 to 2007, he was a researcher at Academic Research Consortium on digital imaging, video, audio and multimedia (CoRIMedia), Canada. His research interests are image processing: image compression, color imaging, image database and searching, medical image analysis, implementing of image processing algorithms on FPGA for real time image analysis. 